\title{
Design of Microstrip Reflective Array Antenna
}

\author{
Meng Li \\ School of Communication Engineering \\ Chengdu University of Information Technology \\ Chengdu, China
}

\begin{abstract}
With the development of communication technology, people have higher and higher requirements for communication quality. Traditional array antennas require complex feed networks and the necessary phase shifters. Conventional reflector antennas are bulky and difficult to manufacture. It is therefore necessary to analyze the traditional characteristics of reflective array antennas to accommodate future adaptability. The microstrip antenna has the advantages of small size, simple structure and small outer shape. Therefore, the working principle and design process of the reflective microstrip array antenna are introduced in detail. A dual-loop antenna operating at $f=4.5 \mathrm{GHz}$ was designed, which simplifies the shape of the antenna and achieves a beam pointing of $30^{\circ}$. Compared with similar literature, the new unit antenna has a simple structure, can realize beam orientation without a phase shifter, can work in the low frequency range of 5G, and has high engineering value.
\end{abstract}

Keywords: Beam orientation; Simple structure; Phase shifter; Microstrip antenna; 5G communication

\section{INTRODUCTION}

With the rapid development of modern microwave communication, satellite communication and 5G technology, parabolic antennas play an increasingly important role[1-3]. However, due to the modern society's requirements for the flexible operation of communication systems, the disadvantages of the traditional parabolic antennas are cumbersome and bulky[4]. The planar array antenna requires a complicated power distribution feed network, a phase shifter, etc., which can easily increase the transmission loss of the antenna and reduce its transmission efficiency[5]. The microstrip reflective array antenna is operated. The microstrip reflective array combines the advantages of a reflective antenna and a microstrip antenna, and is highly valued for its light weight, small size, low price, and ease of manufacture. Different shapes of reflective elements are discussed in the literature of Dahri, M. Hashim. It can be seen from the discussion of various design and architectural features that the reflective arrays of different structures have different phase shifting ranges[6]. However, it can be seen from the text that the shape of the reflec Therefore, this paper designs a micro-band unit with a simple double-ring

structure.tion array unit is complicated, which increases the difficulty of production. The structure is simple, can compensate the phase delay of $0-360^{\circ}$, and can realize the beam orientation function of 30 at $f=4.5 G H z$. It also can be used in $5 \mathrm{G}$ mobile communication systems and other wireless communication systems in the frequency band, and has high engineering practical value.

\section{WORKING PRINCPLE}

The microstrip reflection array is mainly composed of two parts, one part is the feed power source and the other part is a reflective medium plate printed with a large number of microstrip arrays. 


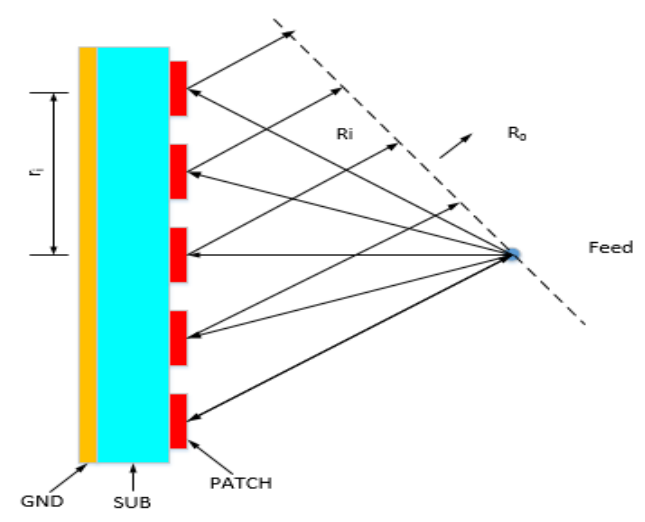

Figure.1 Reflective array antenna working diagram

Working mechanism of microstrip planar reflection antenna: assuming that the planar reflection arrays are all in the far field region of the feed, it can be considered that the electromagnetic waves irradiated to each of the reflection units are plane waves[7]. For a spherical wave, the phase is proportional to the distance between the center of the feed phase and each reflective unit. When the electromagnetic wave radiated by the feed is irradiated from the center of the feed phase to each radiating element on the reflective array, since the transmission distance from the feed to each unit is different, there must be a wave path difference between the respective units, so that each unit is The received incident field has different spatial phase delays, and the size parameters of each unit are reasonably designed according to the phase center of the feed horn and the specified beam pointing, so that it can properly compensate the incident field[8]. From the ray theory, the phase of the ith unit in Figure 1 that needs to be compensated is:

$\phi_{i}=2 \pi \mathrm{N}+k_{0}\left(R_{i}-r_{i} \cdot r_{0}\right) \quad(\mathrm{N}=0,1,2 \ldots)$

$k_{0}$ is the free space wave number, $R_{i}$ is the phase center of the feed to the position of the $i$ th patch, $r_{i}$ represents the position vector from the center of the array to the $i$ th patch、 $r_{0}$ represents the unit vector along the outgoing main beam, $2 \pi N$ indicates that the phase compensation period is $2 \pi$ [8-10].

\subsection{Spatial phase delay unit between array} elements

The spatial phase delay in a planar array reflective antenna is primarily due to the fact that the array peripheral elements are unequal from the center and thus due to differences in the electric field transmission paths.

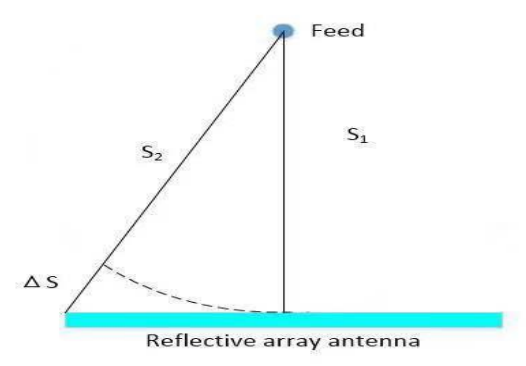

Figure.2 Spatial wave path difference between the units of the reflective array antenna

Therefore, the spatial phase difference that needs to be compensated is :

$$
\Delta \varphi=\Delta S \times 2 \pi / \lambda
$$

Therefore, in the microstrip array design, $\Delta \varphi$ must be compensated first to achieve the same incident field phase of each unit of the array.

\subsection{Analytical method for phase shift characteristics of microstrip reflective array elements}

\subsubsection{Unit antana model}

An isolated unit model, which does not consider the mutual coupling effects of surrounding elements, directly uses plane waves to excite individual isolated elements, and obtains the phase delay generated by electromagnetic waves in the unit according to the phase contrast between the reflected waves and the incident waves. F. Venneri et al. extracted a square 
variable size unit antenna using an isolated unit model. This model is fast calculated by computer, but the drawback is that this analysis method only applies to large cell spacing so that mutual coupling can be ignored.

\subsubsection{Infinite period model}

In the wireless periodic cell model, based on the Floquet theory to simulate the model of the infinite array environment, the influence of the cell spacing on the unit reflection field can be calculated. Therefore, in the unit design, only a single unit model needs to be calculated to complete the wireless large The calculation of the array.

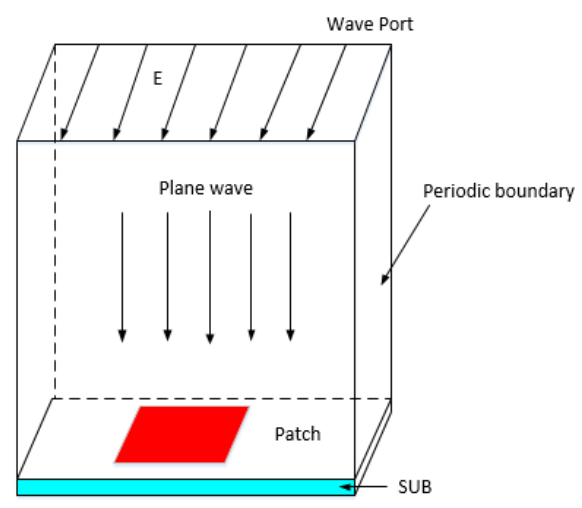

Figure.3 Infinite period model

Similar to the simulation model of a general microstrip antenna, the model mainly consists of three parts, the excitation port, the surrounding periodic boundary and the patch unit. The difference is that the radiation boundary condition in the general model is changed to the periodic boundary condition.

(1) Waveguide Approach-WGA

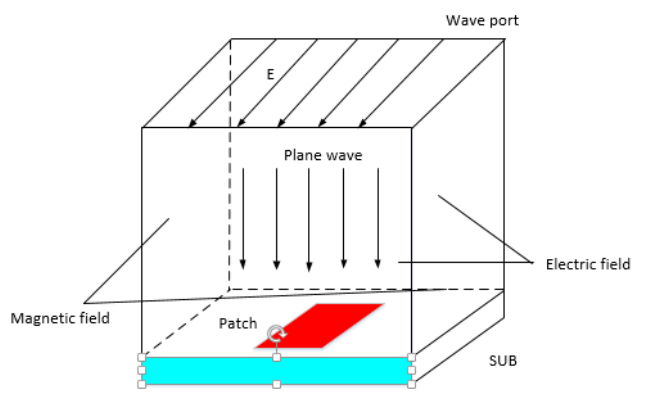

Figure.4 Waveguide simulator model
(2) Master-slave boundary method

The wireless periodic array is simulated by two pairs of master-slave boundaries with a Floquet port. The model excitation port is different from the ordinary wave port by giving two mutually perpendicular electric field excitations to the radiating element on the upper surface of the port, and then porting A layer of PML absorber layer is placed between the master and slave boundaries. This aspect makes up for the simulation flaws that the waveguide simulator can only be used for the normal incidence of plane waves. The master-slave boundary method can illuminate the reflecting unit from any direction, but ensure that the fields on the two master-slave boundaries have the same amplitude and direction.

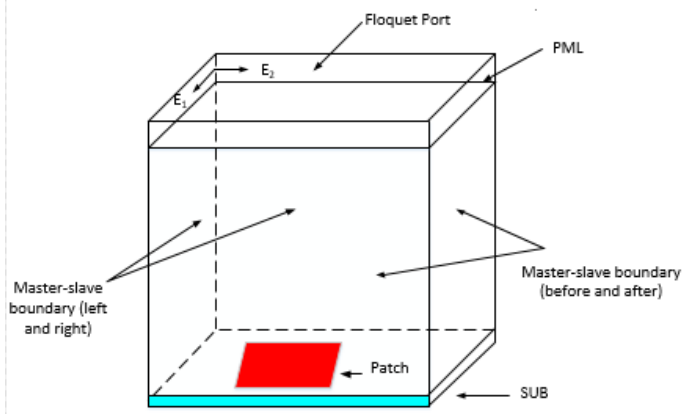

Figure.5 Master-slave boundary method model

\subsection{Several typical phase compensation methods}

During the analysis, the dielectric material, dielectric thickness, and cell spacing remain unchanged.

\subsubsection{Loaded transmission antenna}

Each unit of the patch unit in the array has the same shape and size, but the length of the microstrip line connected to it is different, and the required phase shift is adjusted by changing the length of the microstrip line of each patch in the array.

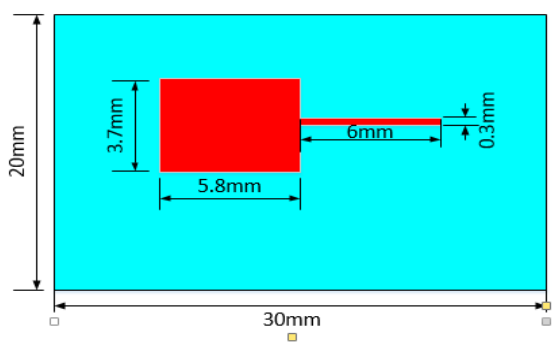




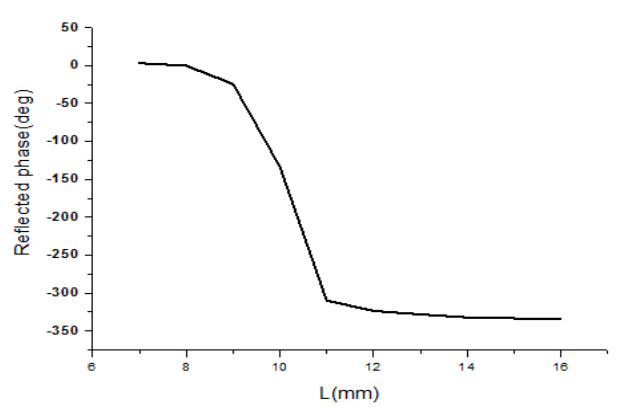

Figure.6 Antenna model and its phase shift range

It is observed from the phase shift curve in the figure that the rectangular patch originating from the load transfer type unit occupies a part of the front surface, so that the phase shift range of the load transfer type unit is very limited $(\Delta=332 \mathrm{deg}$ ).

\subsubsection{Variable size}

The shape of the array consists of reflective elements of the same shape but different sizes, and the appropriate amount of phase shift is provided by adjusting the cell patch size.
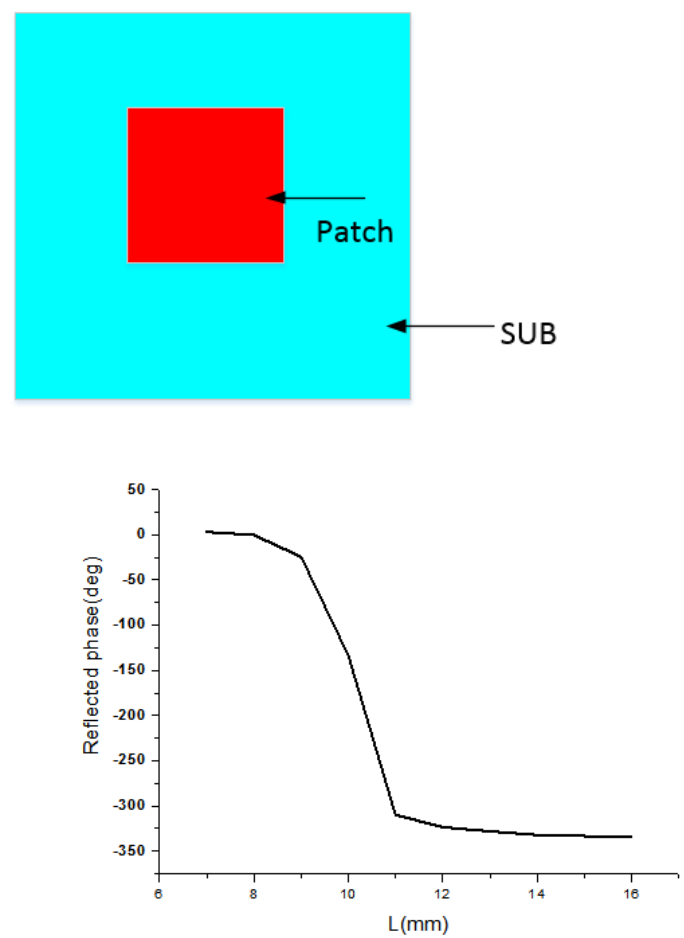

Figure.7 Antenna model and its phase shift range

The phase shifting curve of the square patch unit is close to the "S" shape. It can be seen from the figure that the phase shift range is $327^{\circ}$.

\subsubsection{Slotted unit}

Each patch unit in the array has the same shape and size, and is slotted on the ground plate on the back side. The size of the slot is determined by the amount of phase shift required.
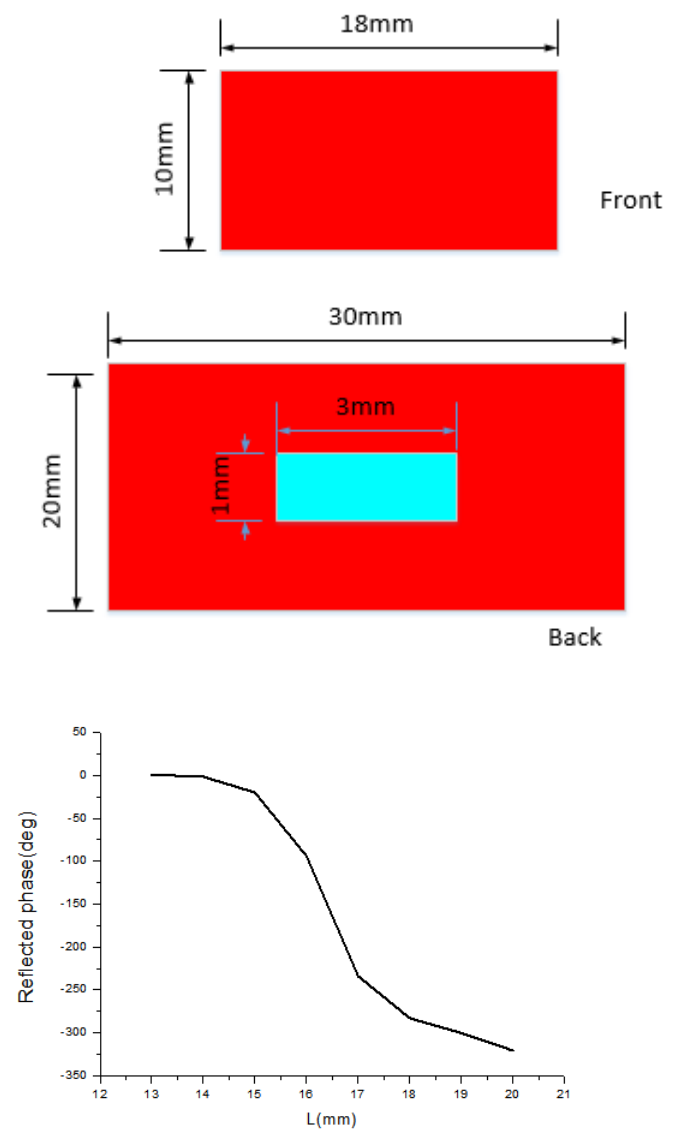

Figure. 8 Antenna model and its phase shift range

As seen in Figure 8, the range of antenna movement is $\Delta=363^{\circ}$

\section{NEW REFLECTVE ARRAY ANTENNA \\ 3.1 reflection unit structure}

A new unit of multi-layer structure is proposed in 
the literature [11], and the phase shift range exceeds $450^{\circ}$. The literature [12]. Proposed a windmill type unit with a phase shift range of $700^{\circ}$. However, the two units are complicated in structure and difficult to manufacture. Therefore, this paper designs a new type of unit with double loop structure, the structure is as follows:
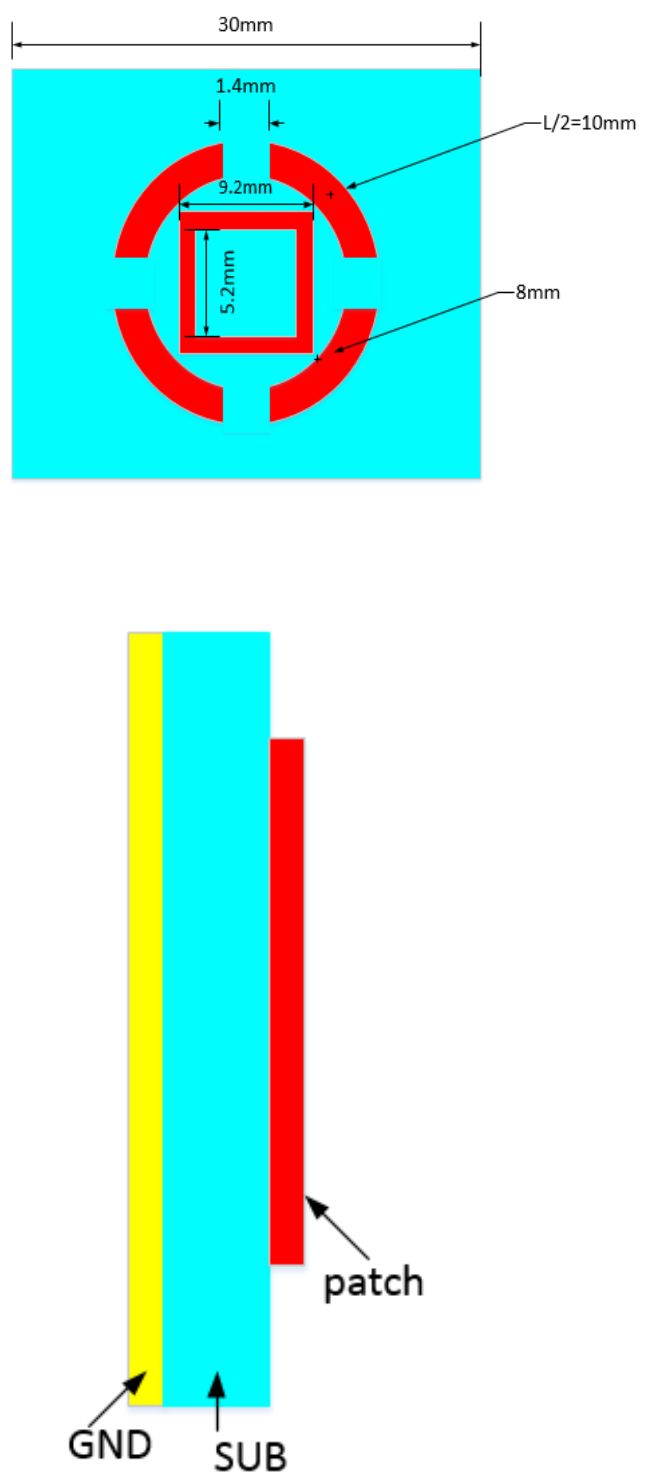

Figure.9 Antenna structure

The unit consists of two annular patches, wherein the inner ring is a square ring, the outer ring is a ring, and there is a gap between the upper and lower sides. Rogers RT/duroid 5880 material with thickness $\mathrm{t}=2 \mathrm{~mm}$, dielectric constant is 2.2 . (Related units are marked in the figure).

\subsection{Influence of unit structure parameters on phase shift characteristics}

(1) Effect of thickness t on phase shifting performance.

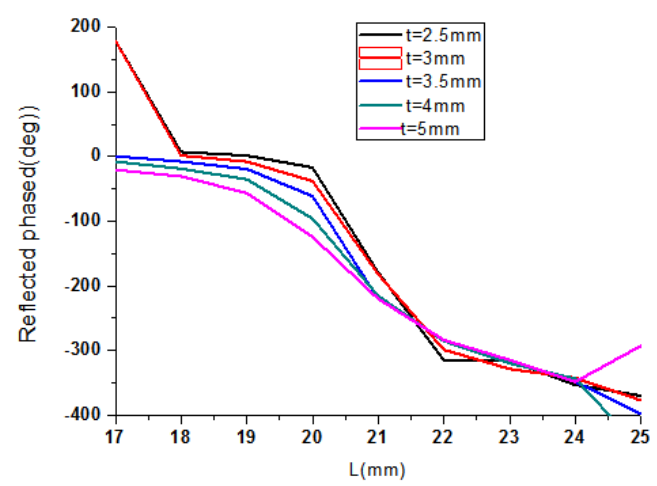

Figure.10 Effect of different parameters $d$ on phase shifting performance

As can be seen from Fig. 10, as the thickness of the substrate increases, the resonance point of the phase shift curve gradually approaches, and the phase shift range is reduced, but still satisfies $360^{\circ}$. Finally take $\mathrm{t}=4 \mathrm{~mm}$.

(2) Effect of parameter d on phase shifting performance

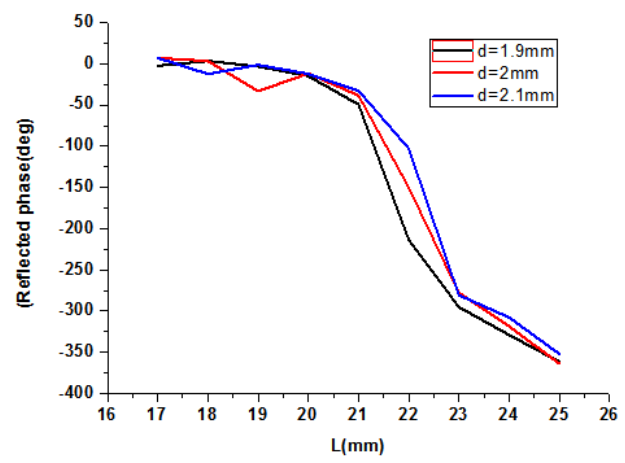

Figure.11 Effect of different parameters $d$ on phase shifting performance

As can be seen from Fig. 11, as the parameter d increases, the outer ring width of the unit antenna increases, the curve becomes steep, and the resonance point distance is zoomed in. Finally, $\mathrm{d}=1.9 \mathrm{~mm}$ is taken.

(3) Effect of parameter g on phase shift 
performance

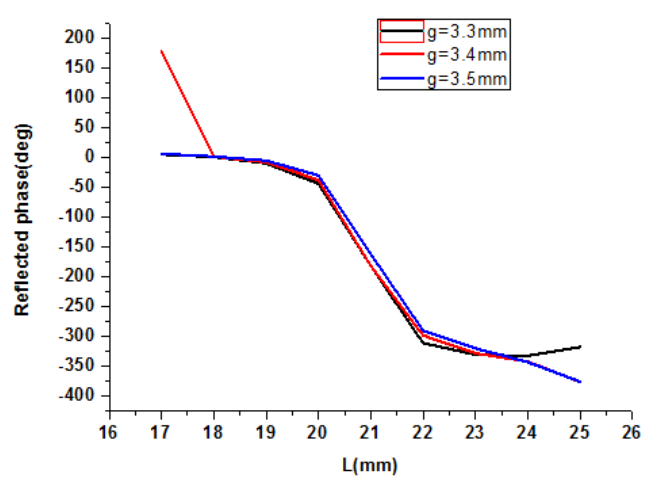

Figure.12 Effect of different parameters g on phase shifting performance

As can be seen from Fig. 12, as the g gradually increases, that is, the interval between the inner and outer rings gradually becomes larger, the phase shift range does not change much. Finally take the height. $\mathrm{g}=3.3$. In summary, the optimized unit parameters are:

\section{Parameters Table 1}

\begin{tabular}{|l|l|l|l|l|l|l|l|}
\hline Variable & $\mathrm{a}$ & $\mathrm{t}$ & $\mathrm{hs}$ & $\mathrm{h}$ & $\mathrm{l}$ & $\mathrm{d}$ & $\mathrm{g}$ \\
\hline length & 30 & 4 & 0.035 & 15 & 20 & 1.9 & 3.3 \\
\hline
\end{tabular}

The optimized curve is shown below:

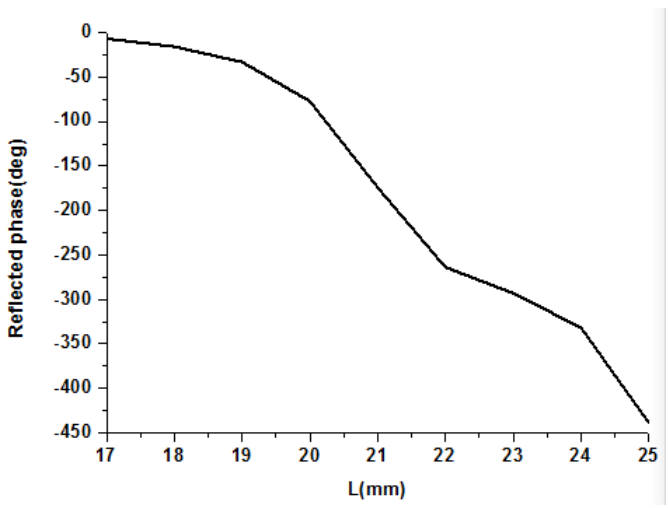

Figure.13 Optimized curve

It can be seen that the phase shift range is $\Delta=395^{\circ}$, greater than $360^{\circ}$, to meet the design requirements.

\subsection{Microstrip reflection array design}

In this paper, the phase shift performance of the new reflection unit is analyzed and analyzed. The phase shift performance is $0 \sim 360^{\circ}$, and the linearity of the phase shift curve is also good. Therefore, the reflecting unit is designed as a reflective array antenna. When the feed is far enough away from the plane array, the feed can be regarded as a plane wave, assuming that the beam points to theta at $30^{\circ}$ and the phase delay of the adjacent array unit is:

$$
\Delta \phi=-\frac{2 \pi f}{c} a \cos \theta
$$

$$
f \text { is the working frequency band, } c \text { is the }
$$
speed of light in vacuum, and $a$ is the spacing between adjacent units.

The array adopts $4 * 4$ array mode, the cell spacing is $\mathrm{a}=12 \mathrm{~mm}$, and the dielectric constant is 2.2 . The beam is directed to theta $=30$ degrees, so the phase of the cells in each row and column needs to be compensated: 
International Journal of Computer Applications Technology and Research

Volume 8-Issue 09, 340-347, 2019, ISSN:-2319-8656

Parameters Table 2
According to Table 2, the corresponding dimensions

of each unit are as follows:

\begin{tabular}{|l|l|l|l|l|}
\hline Column & 1 & 2 & 3 & 4 \\
\hline 1 & & & & \\
\hline 2 & 19 & 21.75 & 22.25 & 23 \\
\hline 3 & 21.75 & 22.25 & 23 & 24.9 \\
\hline 4 & 22.25 & 23 & 24.9 & 21.35 \\
\hline
\end{tabular}

Make a reflection array based on the dimensional data in Table 3.

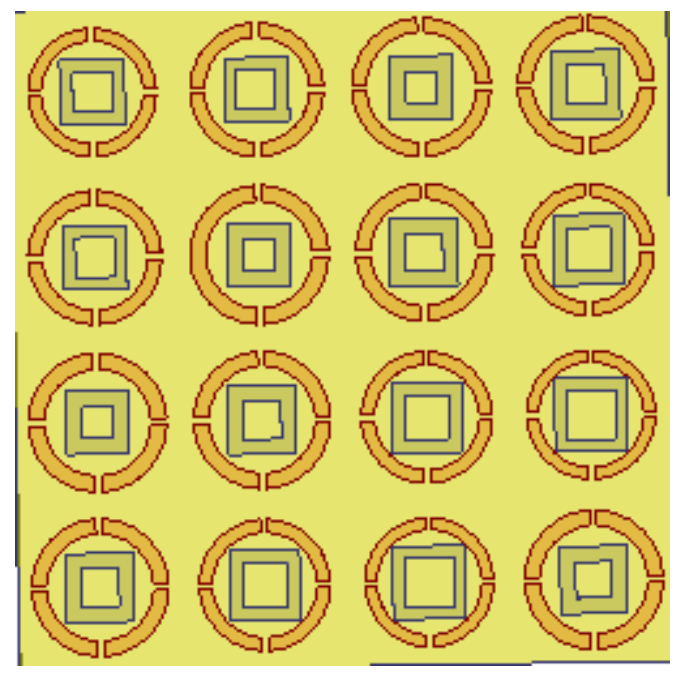

Figure.14 Microstrip reflective array antenna

Simulation and optimization of reflective array antennas using ansoft HFSS software:

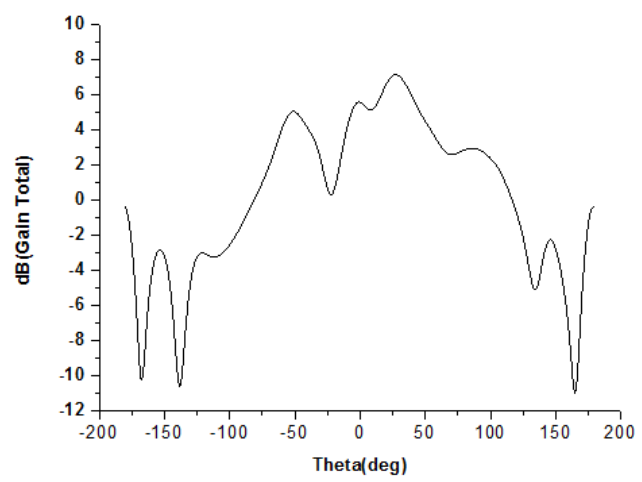

Figure.14 Gain curve of the reflective array

Parameters Table 3

\begin{tabular}{|c|c|c|c|c|}
\hline Column & 1 & 2 & 3 & 4 \\
Row Phase & & & & \\
\hline 1 & -33.24 & -114.24 & -195.24 & -276.24 \\
\hline 2 & -114.24 & -195.24 & -276.24 & -357.24 \\
\hline$\frac{\text { www.ijsea.com }}{3}$ & -195.24 & -276.24 & -357.24 & -438.24 \\
\hline 4 & -276.24 & -357.24 & -438.24 & -519.24 \\
\hline
\end{tabular}


International Journal of Computer Applications Technology and Research

Volume 8-Issue 09, 340-347, 2019, ISSN:-2319-8656

It can be seen from the figure that the pattern of the main beam is in the direction of $30^{\circ}$, which is consistent with the original design, thus verifying that the double-ring unit can achieve beam orientation.

\section{CONCLUSION}

This paper proposes a microstrip reflective array antenna that can be used in the 5G FR1 band, which compensates for the phase shift of the antenna by changing the structure of the antenna unit. The beam pointing angle is set, and the size of the reflective array unit is calculated. Finally, the main beam of the antenna is accurately oriented to a preset $30^{\circ}$ to achieve beam directivity. In the same literature, the unit antenna has a simple structure and is easy to process and design. It can be used in 5G FR1 mobile communication systems and other wireless communication systems, and has high engineering practical value.

\section{REFERENCES:}

[1] Ta, Son Xuat , H. Choo , and I. Park . "Broadband Printed-Dipole Antenna and Its Arrays for 5G Applications." IEEE Antennas and Wireless Propagation Letters PP.99(2017):1-1.

[2] Li, Xichun, et al. "The Future of Mobile Wireless Communication Networks." International Conference on Communication Software \& Networks 2009.

[3] Sharma, Pankaj . "Evolution of Mobile Wireless Communication Networks- $1 \mathrm{G}$ to $5 \mathrm{G}$ as well as Future Prospective of Next Generation Communication Network." International Journal of Computer Science \& Mobile Computing 2.8(2013).

[4] Huang, J. "Analysis of a microstrip reflectarray antenna for microspacecraft application." Tda Progress Report 120(1995).

[5] Chang, Zhuang , et al. "A Reconfigurable Graphene Reflectarray for Generation of Vortex $\mathrm{THz}$ Waves." IEEE Antennas and Wireless Propagation
Letters (2016):1-1.

[6] Dahri, M. Hashim, et al. "A Review of Wideband Reflectarray Antennas for 5G Communication Systems." IEEE AccessPP.99(2017):1-1.

[7] Qin, Pei Yuan , Y. J. Guo , and A. R. Weily . "Broadband Reflectarray Antenna Using Sub-wavelength Elements Based on Double Square Meander-Line Rings." IEEE Transactions on Antennas and Propagation 64.1(2015):1-1.

[8] Chaharmir, M. R. , and J. Shaker . "Design of a broadband, dual-band, large reflectarray using multi open loop elements." Antennas \& Propagation Society International SymposiumIEEE, 2010.

[9] Venneri, F. , S. Costanzo , and M. G. Di . "Bandwidth Behavior of Closely Spaced Aperture-Coupled Reflectarrays." International Journal of Antennas and Propagation2012(2012):1-11.

[10] Li, Qin Yi , Y. C. Jiao , and G. Zhao . "A Novel Microstrip Rectangular-Patch/Ring- Combination Reflectarray Element and Its Application." IEEE Antennas \& Wireless Propagation Letters 8.4(2009):1119-1122.

[11] José A. Encinar. "Design of two-layer printed reflectarray using patches of variable size." IEEE Transactions on Antennas and Propagation 49.10(2001):1403-1410.

[12] Encinar, J. A. , and J. A. Zornoza . "Broadband design of three-layer printed reflectarrays." IEEE Transactions on Antennas and Propagation 51.7(2003):1662-1664. 